\title{
Le monnayage émis À Saittai de Lydie sous Septime Sévère
}

The Coinage Struck at Saittai, Lydia, under Septimius Severus

\section{MICHEL AMANDRY}

Directeur d'Études émérite. École Pratique des Hautes Études, IVe Section. mamandry@noos.fr

\section{RÉSUMÉ:}

La cité de Saittai, en Lydie, a émis, d'Hadrien à Gallien, un monnayage intermittent et de faible ampleur. Les émissions sévériennes, signées par des archontes, ainsi que les séries sans nom d'archonte, sont étudiées ici : un catalogue précis en est établi, une chronologie relative et absolue proposée, un commentaire sur l'onomastique et la typologie donné.

Mots clés: Saittai, Lydie, Septime Sévère et sui, monnayage provincial.

\section{Abstract:}

From Hadrian to the reign of Gallienus, the Lydian city of Saittai produced an intermittent coinage whose output was not abundant. The Severan emissions signed by an archon, together with the series without archon's name, are studied here : a precise catalogue is established, a chronology, relative and absolute, is given, and a commentary on the onomastic and typology is provided.

Key words: Saittai, Lydia, Septimius Severus et sui, provincial coinage.

Copyright: 12017 Michel Amandry. This is an open access paper ditributed under the terms of 
En 2004, l'Université du Missouri faisait l'acquisition d'une monnaie inédite de Saittai, frappée sous Septime Sévère, signée de l'archonte Chariklès ${ }^{1}$. Jane Biers, alors directeur par intérim du Musée d'Art et d'Archéologie, suggéra que Eugene Lane et moi-même publions cette monnaie et $\mathrm{j}$ 'entrai en correspondance avec E. Lane en $2005^{2}$. Sa disparition en 2007 mit fin à ce projet commun, mais je n'avais pas renoncé à la publier. Il me semblait toutefois qu'il fallait insérer cette monnaie dans une étude plus vaste portant sur l'ensemble du monnayage sévérien de Saittai ${ }^{3}$. Pris par d'autres projets, j'ai délaissé ce dossier qui refait surface aujourd'hui, mais j'avais eu la surprise de voir la pièce de Chariklès publiée en $2008^{4}$, la nouvelle équipe à la tête du Musée d'Art et d'Archéologie ignorant manifestement les arrangements passés avec l'ancienne. La publication de P. Lawrence n'a de loin pas épuisé le sujet $^{5}$ et mon travail reste d'actualité ${ }^{\text {. }}$.

Je donne donc d'abord le catalogue ${ }^{7}$ de ce monnayage, avant en bonne méthode de le commenter. En gras les monnaies qui ont été reproduites.

\section{CATALOGUE}

\section{COLLECTIONS PUBLIQUES}

Cambridge: GROSE, S. W. (1929) : Fitzwilliam Museum Catalogue of the McClean Collection of Greek Coins. III. Asia Minor, Farther Asia, Egypt, Africa, Cambridge.

Copenhague: SNG The Royal Collection of Coins and Medals. Danish National Museum. Lydia I. Acrasus-Saïtta, Copenhague, 1947.

Istanbul: GÖKYILDIRIM, T. (2016) : Istanbul Arkeoloji Müzeleri. Lydia Sikkeleri Kataloğu, Istanbul.

Londres: HEAD, B. V. (1902): A Catalogue of the Greek Coins in the British Museum. Catalogue of the Greek Coins in Lydia, Londres.

Munich: LESCHHORN, W. (1997) : SNG Deutschland. Staatliche Münzsammlung München 23. Lydien, Munich.

Paris: BABELON, E. (1898) : Inventaire sommaire de la collection Waddington acquise par l'État en 1897, Paris.

Vienne, Stiftes Schotten: HÜBL, A. (1920) : Die Münzensammlung des Stiftes Schotten in Wien. II. Griechische Münzen, Vienne et Lepzig.
Vienne W. SZAIVERT, W. ; DABURON, C. (2000) : SNG Österreich Sammlung Leypold. Kleinasiatische Münzen der Kaiserzeit. I. Pontus-Lydien, Vienne.

Winterthur: BLOESCH, H. (1997), Griechische Münzen in Winterthur 2, Winterthur.

\section{COLLECTIONS PRIVÉES}

CGT: Collection privée, Malte

Falghera: VISMARA, N. (1992) : Monete provinciale romana. II. Collezione Winsemann Falghera. 3. Septimius Severus - Severus Alexander, Glaux 8, Milan.

Lindgren: LINDGREN, H. C. ; KOVACS, F. (1985) : Ancient Bronze Coins of Asia Minor and the Levant from the Lindgren Collection, San Mateo.

Lindgren III: LINDGREN, H. C. (1993) : Lindgren III. Ancient Greek Bronze Coins from the Lindgren Collection, Quarryville.

Missere: MISSERE, G. ; MISSERE FONTANA, F. (1999) : La collezione Missere di monete romane provinciali, Modène.

Tatiş: TEKIN, O. (2016) : SNG Turkey 10. The Yavuz Tatiş collection. Part 1. Ionia and Lydia, Istanbul.

von Aulock: VON AULOCK, H. (1963) : SNG Deutschland. Sammlung v. Aulock 8. Lydien, Berlin; VON AULOCK, H ; FRANKE P. R. (1968) : SNG Deutschland. Sammlung v. Aulock 17. Nachträge III. Ionien. Karien. Lydien, Berlin.

Weber: FORRER, L. (1926) : Descriptive Catalogue of the Collection of Greek Coins Formed by Sir Hermann Weber M. D. 1823-1918. III. Asia, Londres.

\section{A. ÉMISSIONS AVEC NOM D’ARCHONTE}

\section{Atticus premier archonte pour le deuxième fois}

\section{Septime Sévère}

$$
\text { 1. AE. 33-35 mm, 26,83 g (4). Axe : } 6
$$

$\mathrm{AY} \cdot \mathrm{K} \bullet \Lambda \bullet \mathrm{C}-\mathrm{E} \Pi \cdot \mathrm{CEOYH}-\mathrm{POC} \Pi$; buste lauré et cuirassé de Septime Sévère, à dr., avec paludamentum, vu de trois-quarts en arrière

EПI AT - TIKO - Y•AP•A $\bullet$ TO B ; CAITTH/N $\Omega N$ à l'exergue ; Asclépios debout à dr., regardant vers la g., 
sa main dr. appuyée sur un bâton autour duquel est enroulé un serpent ; Hygie debout à g., regardant à dr., tenant une phiale dans laquelle se nourrit un serpent; entre eux, Télesphore debout de face.

1. P FG 1064 (= Waddington $5175=$ Kraft Taf. 77, $\left.2 \mathrm{a}^{8}\right)$, 29,$40 ; 2$. Winterthur $3891,26,02 ; 3$. Berlin 471/1883, 24,17; 4. Numismatica Genevensis 5, 3 Déc. 2008, lot 265 (ex Leu 50, 25 Avril 1990, lot 330), 27,74
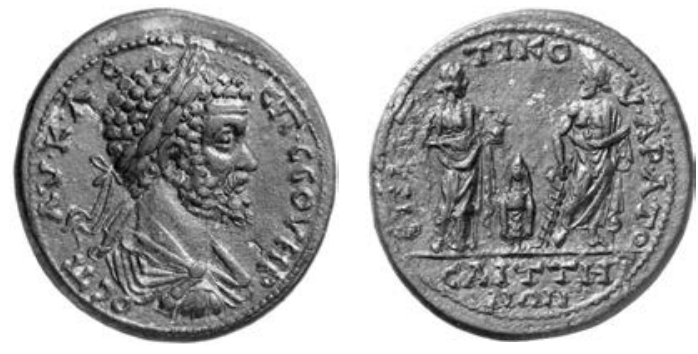

NB. La monnaie publiée par F. Imhoof-Blumer, RSN VI [1896], p. $280, \mathrm{n}^{\circ} 8(=L S 129 / 8)$ indiquée comme appartenant à sa collection ne se trouve pas à Berlin.

\section{Julia Domna}

\section{AE. 28-29 mm, $13,70 \mathrm{~g}(4)$. Axe : 6}

IOY $\Lambda$ IA $\Delta \mathrm{O}$ - MNA CEBACT; buste drapé de Julia Domna, à dr.

EПI ATTIKOY - APX A TO•B CAI; TTHN $\Omega N$ à l'exergue ; temple tétrastyle avec arcature à l'intérieur duquel se trouve la statue d'Aphrodite Cnidia.

1. Hauck et Aufhäuser 18, 5-6 Oct. 2004, lot 489, 12,50 ; 2. VAuctions London Coin Galleries Auction 3, 10 Nov. 2016, lot 55, 15,56 ; 3-4. Collection CGT, 14,07, 12,68
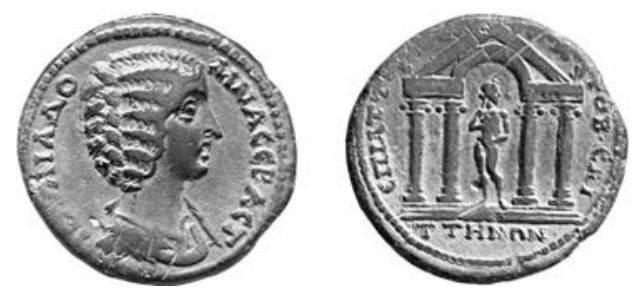

3. AE. 28-29 mm, 13,71 g (4). Axe : 6

IOYAIA $\Delta \mathrm{O}$ - MNA CEBACT; buste drapé de Julia Domna, à dr.

EПI ATTIKOY A - PX A TO•B CA; I/T//TH/N// $/ \mathrm{N}$ dans le champ, à g. et à dr., de bas en haut; Héraclès nu de face, regardant à dr., la main dr. posée sur sa massue, la peau de lion sur son bras $g$.

1. Paris FG $1068^{9}$ (= Kraft Taf. $\left.79,21 \mathrm{e}^{10}\right), 12,29 ; 2$. Lindgren 792, 14,53 ; 3. Gorny et Mosch 130, 8 Mars 2004, lot 1852 (ex Gorny et Mosch 125, 13 Oct. 2003, lot 313), 14,99 ; 4. Collection CGT, 13.03
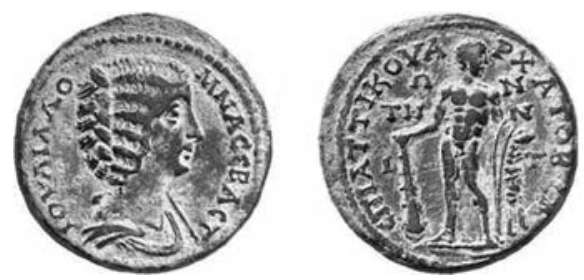

Monnaies sans portrait impérial

4. AE. $23 \mathrm{~mm}, 6,55 \mathrm{~g}(23)$. Axe : 6

$\Delta$ HMOC - CAITTHNQN ; tête laurée du Dèmos, à dr. EПI ATTIKOY - APX A TO B ; Cybèle assise à g., coiffée du polos, tenant une phiale dans sa main dr. tendue, son bras g. posé sur un tambourin ; à ses pieds, un lion.

1. Londres BMC 9 (et Pl. 23, 3 = Martin Saïttai 3/2), 8,36 ; 2. Londres BMC 10, 7.61 (= Martin, Saïttai $3 / 3$; 3. Paris FG 1054 (= Waddington $5167=$ Martin Saïtai $3 / 7$ et 3/22 $2^{11}$ ), 6,92 ; 4. Paris Delepierre 1966.453 (Martin Saïttai 3/-), 5,91 ; 5. Istanbul 391, 4,71 (Martin Saïttai 3/-) ; 6. New York 1944.100.49362, 7.02 (Martin Saïttai 3/-) ; 7. CNG EA 255, 4 Mai 2011, lot 232 (Martin Saïttai 3/18 ${ }^{12}$ ), 5,43; 8. Collection CGT (ex CNG EA 226, 27 Janvier 2010, lot 439) (Martin Saïttai 3/4), 7,47 ; 9-12. Collection CGT, 8,02 , 7,78, 5,91, 5,39 ; 13-29. Voir Martin Saïttai 3/1, 5-6, 8-17, 19-21, 23
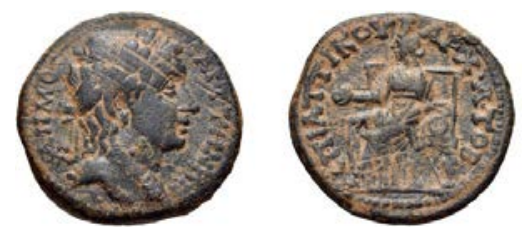


\section{AE. $23 \mathrm{~mm}, 6,32 \mathrm{~g}(2)$. Axe : 6}

$\Delta$ HMOC - CAITTHN $\Omega N$; tête laurée du Dèmos, à dr. EПI ATTIKOY - APX A TO B ; Apollo nu debout à dr., tenant le plectrum de sa main dr., la lyre posée sur son bras $g$.

1. Londres BMC 8 (= Martin Saïttai 4/1), 6,14 ; 2. Collection CGT, 6.49 .
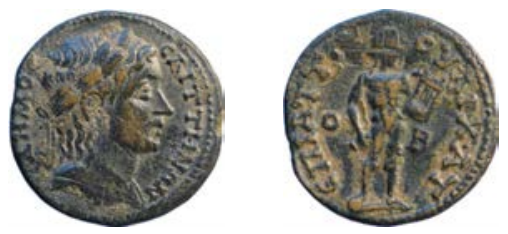

II. Andronikos premier archonte

Septime Sévère

1. AE. $24 / 25 \mathrm{~mm}, 8,93 \mathrm{~g}(21)$. Axe : 6

AY KAI $\Lambda$ CEП - CEOYHPOC ПEP; tête laurée de Septime Sévère, à dr.

EПI AN $\triangle$ PONEIKOY APX A CAITTH, dans le champ, à g. et à dr., N- $\Omega N$ (ou - CAIT, dans le champ, à g. et à dr., T-H/N- $\Omega$ N ou - CAITT, dans le champ, à g. et à dr., $H N-\Omega N$ ); Mên debout à g., un croissant derrière ses épaules, vêtu d'un chiton court, d'un himation noué autour de ses épaules, de bottes et d'un bonnet phrygien, tenant dans sa main dr. tendue une pomme de pin, sa main g. reposant sur un sceptre vertical se terminant en forme de pomme de pin.

1. Paris FG 1065 (= Waddington 5176), 8,91; 2. Londres BMC 42 (et pl. XXIII, 8), 6,73; 3. Londres BMC 43 (= Kraft Taf. 69, 50a) ${ }^{13}, 8,83$; 4. Londres 1979-1-1-2017 (ex von Aulock SNG 3098), 9,62; 5. Munich (Cousinery) SNG 441,9,39; 6. Berlin 8049, 8,03; 7. Berlin I-B, 10,26 ; 8. Berlin v. Knobelsdorf, 7,20 ; 9. Vienne GR 30153, 8,$12 ; 10$. Vienne Leypold SNG 1162, 10,34; 11 . Vienne Stiftes Schotten 3528, 8,84; 12. SNG Turkey 10 Tatiş 709, 10,85 ; 13. Lindgren A790C, 9,68; 14. Lindgren 1343 , 9,95; 15-16. CGT, 8,10 (ex Naville Numismatic 22, 1 Mai 2016, lot 262), 8,09; 17. Peus 366, Burstein, 29 Oct. 2000, lot 725, 9,25; 18. Gorny et Mosch 134, 11 Oct. 2004, lot 1980 (ex Gorny et Mosch 108, 2001, lot 1497), 8,$49 ; 19$. VCoins T. Vossen $5147,8,72 ; \mathbf{2 0 . C N G}$
EA 350, 6 May 2015, lot 352, 9,22 ; 21. Emporium Hamburg 74, 12 Nov. 2015, lot 256, 8,83.
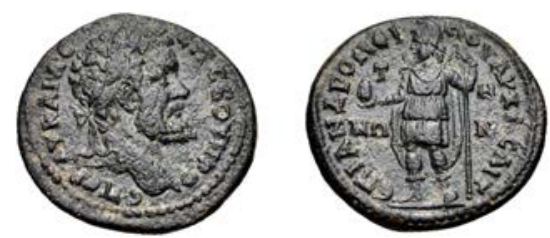

2.AE.24/25 mm, 7,71 g (11). Axe: 6

Même description

EПI AN $\triangle$ PONEIKOY APX A CAITTH, dans le champ, à g. et à dr., $\mathrm{N} \Omega \mathrm{N}$; Tychè debout à g., coiffée du calathos, tenant gouvernail et corne d'abondance.

1. Paris FG 1066 (= Waddington 5177), 7,65; 2. Paris FG 2929 (monnaie classée à Téos), 6,58 ; 3. Paris AA.GR.815 (ex Waddington), 8.21 ; 4. Londres BMC 41, 8,$22 ; 5$. Copenhague SNG 404, 8,67; 6. Vienne GR $31977,8,56$; 7. SNG Turkey 10 Tatiş 708, 8,49 ; 8-9. Istanbul 406-7, 6.39, 7.60 ; 10. Lindgren III, 509, 6,46 ; 11. Collection CGT, 8,03 .
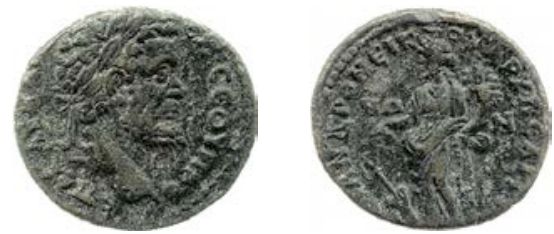

3. AE. $23 \mathrm{~mm}, 6,73 \mathrm{~g}(8)$. Axis : 6

Même description

EПI ANAPONEIKOY APX A CAITT, dans le champ, à g. et à dr., HN- $\Omega \mathrm{N}$; Apollo nu debout à dr., tenant le plectrum de sa main dr., la lyre posée sur son bras $g$.

1. Paris 1067B, 6.89 ; 2. Berlin I-B, 8.12 ; 3. Falghera 1347, 6.01 ; 4. CGT, 6.06 ; 5. Vienne Leypold SNG 1160, 5,83 ; 6. Yale University Art Gallery 2004.6.2931, 6.44 ; 7. Solidus Numismatik e.k., Monthly Auction 19, 23 Septembre 2017, lot 474 (partie du lot) (ex Solidus Online-Auktion 15, 20 Mai 2017, lot 144, partie du lot), 8.45 ; 8. Collection CGT, 6,06. 

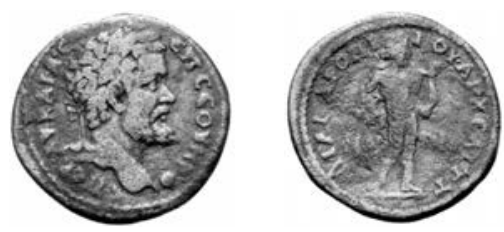

\section{Julia Domna}

4. AE. 34/35 mm, 24,23 (6). Axe: 6

IOYAIA - CEBACTH; buste drapé de Julia Domna, à dr. EПI•AN $\triangle$ PONEIKOY APX A CAITT, dans le champ, à g. et à dr., HN $\Omega N$; Mên debout à g., un croissant derrière ses épaules, vêtu d'un chiton court, d'un himation noué autour de ses épaules, de bottes et d'un bonnet phrygien, tenant dans sa main dr. tendue une pomme de pin, sa main g. reposant sur un sceptre vertical se terminant en forme de pomme de pin.

1. Paris 1069 (= Waddington 5179), 20,22; 2. Londres 1901-7-4-8, 27,98; 3. Munich SNG 443, 26.20; 4. Cambridge McClean 8705 (et pl. 305.14), 24,80; 5. Vienne GR 19521, 25,35 ; 6. Hirsch XXI, Consul Weber, lot 3372; 6. Prowe (=T. Prowe, Quelques monnaies grecques d'Asie mineure, 1912, p. 5, n² 23. Monnaie classée à Adramytion. L'attribution correcte est due à I-B, SNR 19, 1913, p. 56) ; 7. CGT, 20,82.
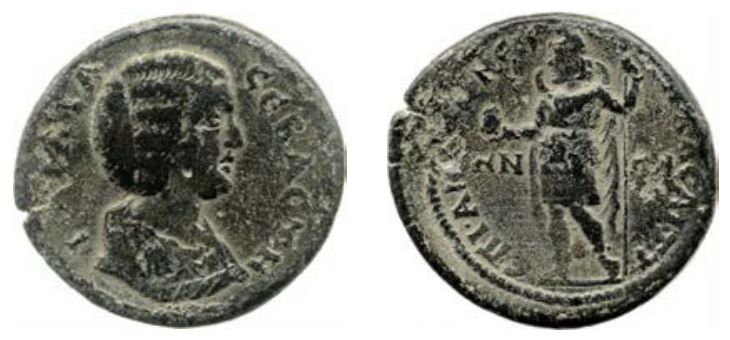

\section{Clodius Albinus César}

5. AE. $28 \mathrm{~mm}, 11,33 \mathrm{~g}(3)$. Axe : 6

$\mathrm{K} \Lambda \Omega$ CEП・ - •A $\Lambda$ BEINOC K - AICAP; tête nue de Clodius Albinus, à dr.

EПI ANDPONEIKOY APX A CAITTH, dans le champ, à g. et à dr., $N \Omega \mathrm{N}$; Zeus Lydios debout à g., tenant un aigle sur sa main dr. tendue, sa main $\mathrm{g}$. reposant sur un sceptre vertical.
1. Berlin Löbb., 14,12 ; 2. CGT, 7,87 ; 3. Boston MFA 1972.858 (ex MM 41, 18-19 June 1970, lot 431 14), 12,00
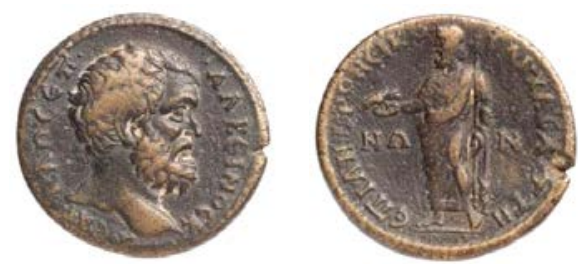

6. AE. $28 \mathrm{~mm}, 12,35 \mathrm{~g}(8)$. Axe : 6, 12 (1)

Même description

EПI AN $\triangle$ PONEIKOY APX A CAITT, dans le champ, à g. et à dr., HN $\Omega N$; Aphrodite Cnidia, debout à g., nue, se couvrant pudiquement; à g., Eros debout à dr., tenant une torche ; à dr., dauphin plongeant.

1. Munich $76158=$ SNG 440, 12,$15 ; 2$. Berlin I-B $(=$ RSN VI, 1896, p. $280, \mathrm{n}^{\circ} 7=$ Lydische Stadtmünzen, 1897, p. 129, n 7), 13,61; 3. Istanbul 404, 14.43 ; 4. CNG MBS 57, 4 April 2001, lot 810 (ex CNG MBS 54, 14 Juin 2000, lot 1110), 10,16 ; 5. MM Deutschland 15, Righetti 4, 21-22 Oct. 2004, lot 815 (ex Bankhaus Aufhäuser 6, 5-6 Oct. 1989, lot 441; MM Numismatics Ltd I, 7 Dec. 1997, lot 203), 12,03 ; 6. Jacquier Auktion 37, 7 Sep. 2012, lot 314 (ex Aufhaüser 13, 7-8 Oct. 1997, lot 408), 11,39; 7. Gitbud et Naumann Auction 12, 2 Feb. 2014, lot 386, 12,$60 ; 8$. NAC 80,20 Oct. 2014 , lot $125,12,41$.
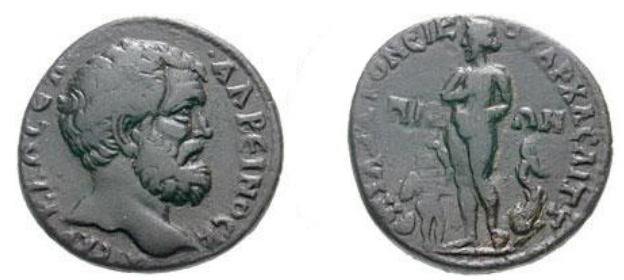

7. AE. $28 \mathrm{~mm}, 11,21 \mathrm{~g}(3)$. Axe : 12 (2)

Même description

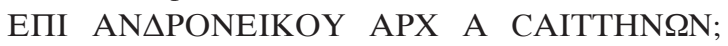
temple tétrastyle à l'intérieur duquel se trouve la statue d'Aphrodite Cnidia.

1. Londres 1973-1-4-8, 9,72; 2. Istanbul 405, 11,42; 3 . Gorny et Mosch 211, 4 March 2013, lot 485, 12,49. 

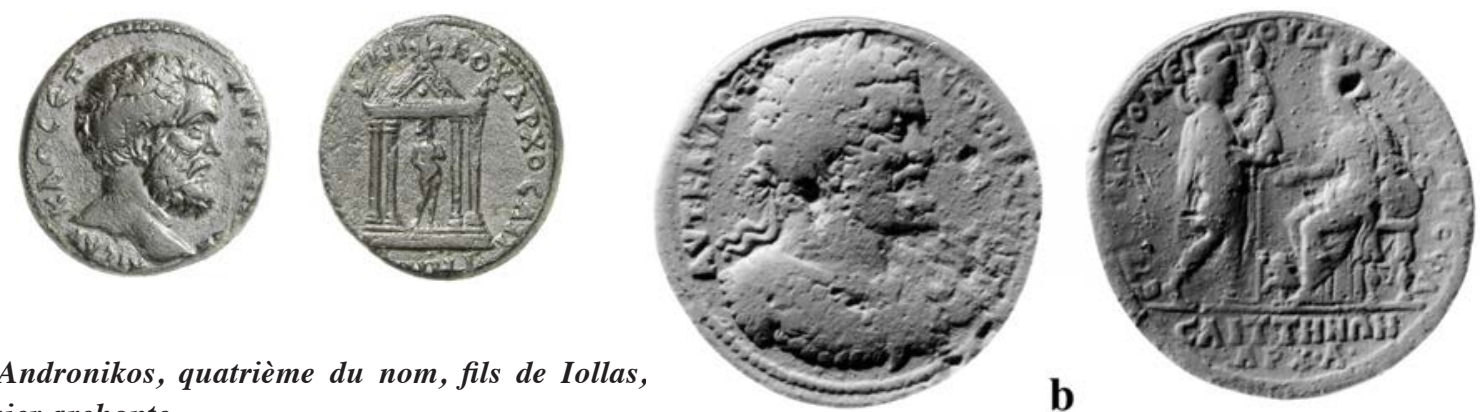

III. Andronikos,
premier archonte

\section{Septime Sévère}

1. AE. $45 \mathrm{~mm}, 44,29 \mathrm{~g}(3)$. Axe : 6

$\mathrm{AYT} \bullet \mathrm{KAI} \bullet \Lambda \bullet \mathrm{CE} \bullet-\mathrm{CEOYHPOC} \mathrm{ПЕРTI;} \mathrm{buste}$ lauré et cuirassé (avec gorgoneion) de Septime Sévère, à dr.

EПI AN $\triangle \mathrm{PONEIKOY} \bullet \Delta \bullet \mathrm{IO} \Lambda \Lambda \mathrm{A} \bullet \mathrm{K} \bullet \mathrm{CTE} \Phi \mathrm{A}$; CAITTHN $\Omega N / A P X \bullet A \bullet$ à l'exergue ; Cybèle assise à $\mathrm{g}$., portant une couronne à tours crénelées, tenant une phiale dans sa main dr. tendue, son bras g. posé sur un tambourin ; à ses pieds, un lion. En face d'elle, Mên debout à dr., un croissant derrière ses épaules, vêtu d'un chiton court, d'un himation noué autour de ses épaules, de bottes et d'un bonnet phrygien, tendant de sa main dr. une pomme de pin à Cybèle, sa main $g$. reposant sur un sceptre vertical se terminant en forme de pomme de pin.

1. B B-I 1928 (= RSN 19 [1913], p. 56, n 161), 40,48; 2. Winterthur 3889 (a), 51,64; 3. NY 1973.191.14 (b), 40,74 .

Les trois exemplaires de la même paire de coins.
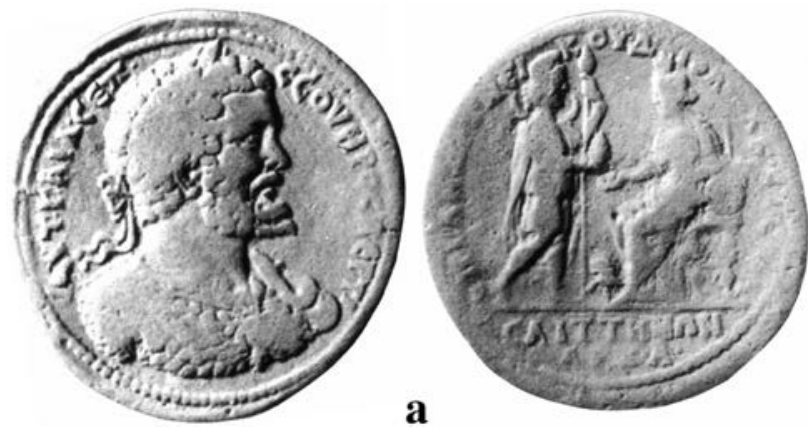

$\mathbf{a}$
Julia Domna

\section{AE. 39-40 mm, 32,73 g (1). Axe :?}

IOY $\Lambda$ IA - CEBACTH; buste drapé de Julia Domna, à dr. $\mathrm{E \Pi I} \bullet \mathrm{AN} \Delta \mathrm{PONIKOY} \bullet \Delta \cdot \mathrm{IO} \Lambda \Lambda \mathrm{A} \quad \mathrm{K} \quad \mathrm{CTE} \Phi$; $\mathrm{APX} \bullet \mathrm{A} \bullet$ dans le champ à g.; CAITTHN $\Omega / \mathrm{N} /$ à l'exergue ; Cybèle assise à g., coiffée du polos, tenant une phiale dans sa main dr. tendue, son bras g. posé sur un tambourin ; à ses pieds, un lion.

1. Peus 366, coll. Marcel Burstein, 25 Oct. 2000, lot 726, 32,73 .
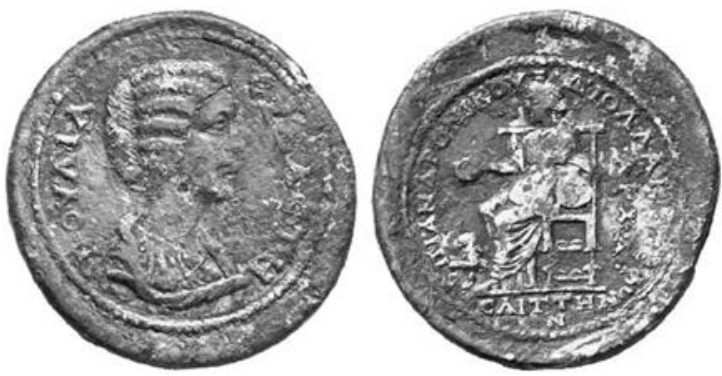

\section{Chariclès premier archonte pour le deuxième fois}

Septime Sévère

1.AE. $45 \mathrm{~mm}, 51,04 \mathrm{~g}(1)$. Axe : 6

AY KAI $\Lambda$ CE[ПT] - CEOYHPOC ПE; buste lauré et cuirassé de Septime Sévère, à dr., avec paludamentum, vu de trois-quarts en arrière

EПI COC XA - PIK $\Lambda$ E - OYC APX A $\underline{\text { TO}} \cdot \mathrm{B}$; CAITTHN $\Omega / \mathrm{N}$ à l'exergue ; Cybèle assise à g., portant une couronne à tours crénelées, tenant une phiale dans sa main dr. tendue, son bras g. posé sur un tambourin ; à ses 
pieds, un lion. En face d'elle, Mên debout à dr., un croissant derrière ses épaules, vêtu d'un chiton court, d'un himation noué autour de ses épaules, de bottes et d'un bonnet phrygien, tendant de sa main dr. une pomme de pin à Cybèle, sa main g. reposant sur un sceptre vertical se terminant en forme de pomme de pin; au pied du sceptre, petit personnage dont l'identification est incertaine.

1. Université du Missouri, Musée d'Art et d'Archéologie 2004.5 ${ }^{15}$ (ex Kovacs MBS XV, 1 Oct. 2003, lot 244), 51,04.
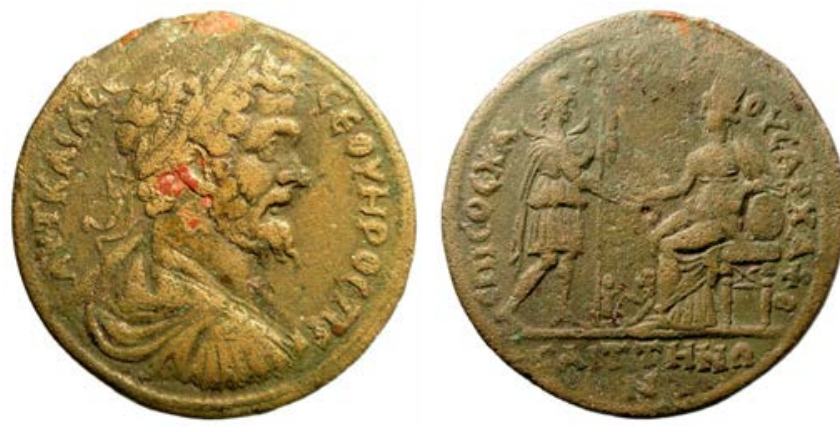

\section{Julia Domna}

2. AE. 35-36 mm, 22,13 g (1). Axe : 6

IOYAIA - CEBACTH; buste drapé de Julia Domna, à dr.

EIПI (sic) COC XAP - IKAEOYC APX A TO B ; CAITTH/NQN à l'exergue ; l'empereur en habit militaire au galop à cheval à dr., son paludamentum flottant dans son dos, tenant de sa main dr. une lance qu'il s'apprête à jeter sur un ennemi à-demi agenouillé à dr.

1. Winterthur G 3893 (= I-B, RSN XIV [1908], p. 16-17 = I-B, Zur Griechischen und Römischen Münzkunde, $\mathrm{p}$. 128-9, Saïtta 1), 22,13.
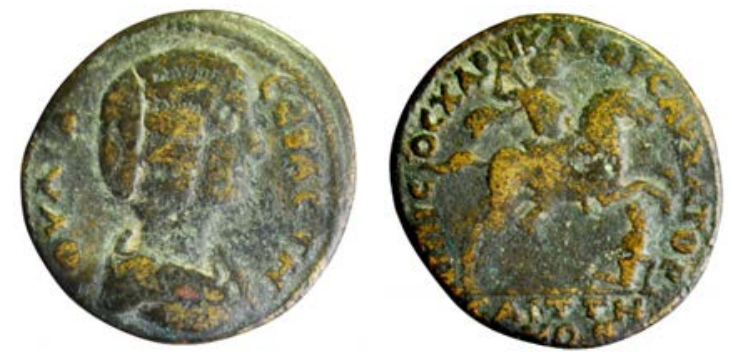

3. AE. 35-36 mm, 23,66 g (1). Axe :?

IOYAIA - CEBACTH; buste drapé de Julia Domna, à dr.

EПI COC XA - PIKA - EOYC APX A TO B ; CAITTH/N $\Omega N$ à l'exergue ; Asclépios debout à dr., regardant vers la g., sa main dr. appuyée sur un bâton autour duquel est enroulé un serpent ; Hygie debout à g., regardant à dr., tenant une phiale dans laquelle se nourrit un serpent ; entre eux, Télesphore debout de face.

1. Pecunem Gitbud et Naumann 34, 2 Août 2015, lot 681, 23,66.
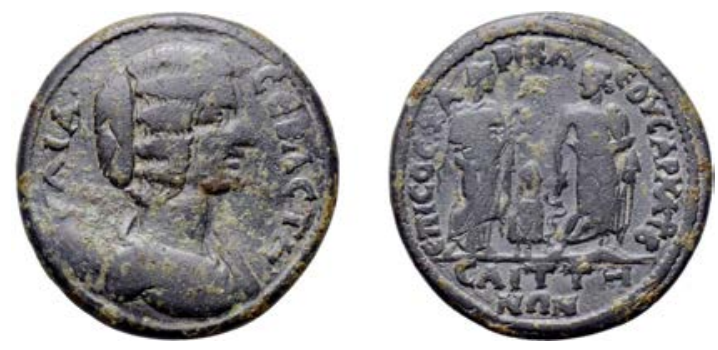

\section{Caracalla Auguste}

4. AE. 35-36 mm, 23,40 g (1). Axe : 6

AY K M AY - ANTSNEINOC; buste lauré et cuirassé de Caracalla à dr., avec paludamentum, vu de trois-quarts en arrière

EПI COC XAP - IKAEOYC APX A TO B ; CAITTHN $/ \mathrm{N}$ à l'exergue ; l'empereur en habit militaire au galop à cheval à dr., son paludamentum flottant dans son dos, tenant de sa main dr. une lance qu'il s'apprête à jeter sur un ennemi à-demi agenouillé à dr.

1. Missere 384, 23,40.
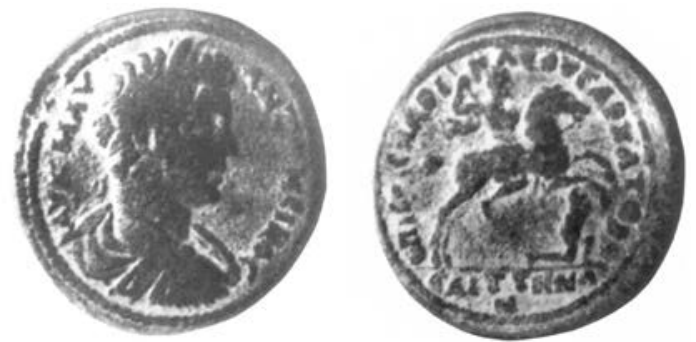


\section{AE. 35-36 mm, 22,26 g (4). Axe : 12}

AY K M AY - ANTQNEINOC; buste lauré et cuirassé de Caracalla à dr., avec paludamentum, vu de trois-quarts en arrière

EПI COC XA - PIKA - EOYC•APX A TO B ; CAITTH/N $\Omega N$ à l'exergue ; Asclépios debout à dr., regardant vers la g., sa main dr. appuyée sur un bâton autour duquel est enroulé un serpent ; Hygie debout à g., regardant à dr., tenant une phiale dans laquelle se nourrit un serpent; entre eux, Télesphore debout de face.

1. Paris FG 1077 (= Waddington 5185), 15,87 ; 2. B Löbb. (= ZfN 12 [1885], p. 338, 3), 27,03; 3. MM Deutschland 32, 26 Mai 2010, lot 245 (ex MM 41, 18 19 Juin 1970, lot 432 ; The New York Sale XI, 11 Janv. 2006, lot 204), 26,78 ; 4. Gorny et Mosch 191, 11 Oct. 2010 , lot $1807,19,35$
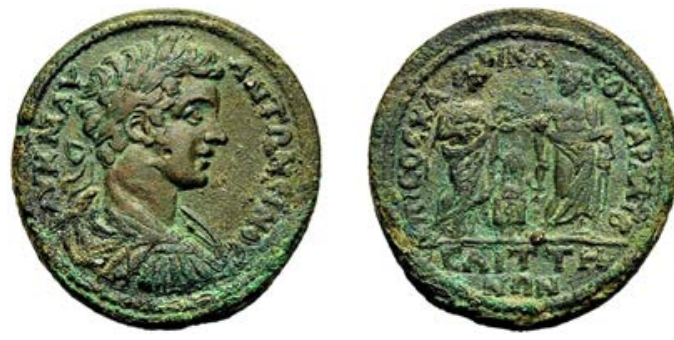

3. Probablement même coin de revers que Julia Domna IV, 3, 1 .

\section{AE. $28 \mathrm{~mm}, 14,88 \mathrm{~g}(3)$. Axe : 6}

$\mathrm{AY} \bullet \mathrm{K} \bullet \mathrm{M} \bullet \mathrm{AY} \bullet-\mathrm{ANT} \Omega N E I N O C$; buste lauré et cuirassé de Caracalla à dr., avec paludamentum, vu de troisquarts en arrière

EПI COC XAPIK $\Lambda$ E - OYC AP(sic) A TO B CAITT; dans le champ, à g. et à dr., $\mathrm{H} / \mathrm{N} / / \Omega / \mathrm{N}$ à l'exergue ; Héraclès nu de face, regardant à dr., la main dr. posée sur sa massue, la peau de lion sur son bras $\mathrm{g}$.

1. Vienne GR 37024, 16,37 ; 2. CNG EA 352, 3 Juin 2015, lot 307, 16,85; 3. Collection CGT, 11,43 (légende de droit et de revers retouchés).
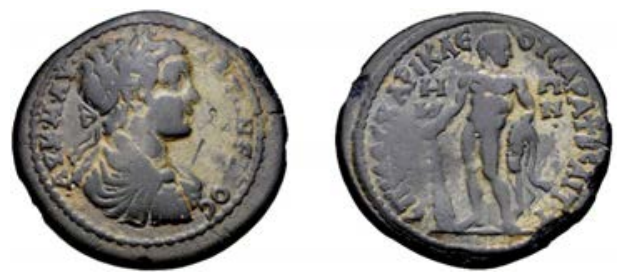

7.AE. $28 \mathrm{~mm}, 13,69 \mathrm{~g}(6)$. Axe : 6 (4), 12 (2)

$\mathrm{AY} \bullet \mathrm{K} \bullet \mathrm{M} \bullet \mathrm{AY} \bullet-\mathrm{ANT} \Omega N E I N O C$; buste lauré et cuirassé de Caracalla à dr., avec paludamentum, vu de troisquarts en arrière

EПI COC XAPIK $А E$ - OYC APX A TO B CAI; TTHNQN à l'exergue ; temple tétrastyle à l'intérieur duquel se trouve la statue d'Aphrodite Cnidia.

1. Paris FG 1076 (= Waddington 5184), 15,14; 2. Londres BMC 50 (= Kraft Taf. 70, 58b) ${ }^{16}, 13,69$; 3. Londres BMC 51, 13,12; 4. Vienne Leypold SNG 1168, 10,92 ; 5-6. Collection CGT, 16,15, 13,08.
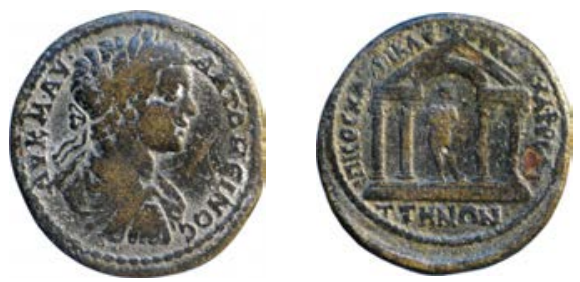

\section{Géta César}

8.AE. $40 \mathrm{~mm}, 33,04 \mathrm{~g}(1)$. Axe : 12

$\Lambda$ СЕПТ - ГЕТАС CE; buste tête nue et cuirassé de Géta à dr., avec paludamentum, vu de trois-quarts en avant

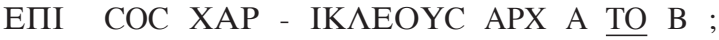
CAITTHN/ $\Omega N$ à l'exergue ; Héraclès nu de face, regardant à dr., tenant de sa main dr. sa massue levée derrière sa tête, la peau de lion sur son bras g. ; derrière sa jambe dr., carquois et arc ; à ses pieds, Géryon terrassé, levant les deux mains en signe de supplication.

1. F. Schulman, The Thomas Ollive Mabbott coll., I, New York, 6-11 Juin 1969, lot 1831 (ex J. Hirsch XIII, Rhousopoulos, 15 Mai 1905, lot 4063) ; 2. Falghera 1348, 33,04.
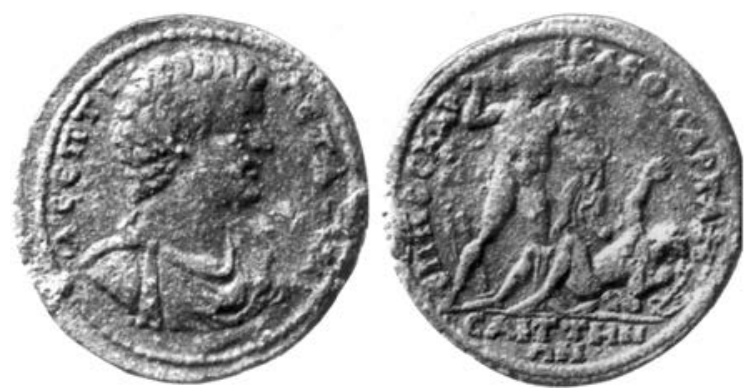
Monnaies sans portrait impérial ${ }^{17}$

\section{AE. 20-21 mm, 6,12g (3). Axe : 6, 12}

IEPA CYN - KAHTOC ; buste drapé du Sénat, à dr. EПI COC XAPI - KAEOYC AP CAITTHNQ; N dans le champ g.; Cybèle assise à g., coiffée du polos, tenant une phiale dans sa main dr. tendue, son bras $\mathrm{g}$. posé sur un tambourin; à ses pieds, un lion.

1. Paris FG 1044A, 5,70 ; 2-3. Londres BMC 20-1, 6,51, 6,16 .
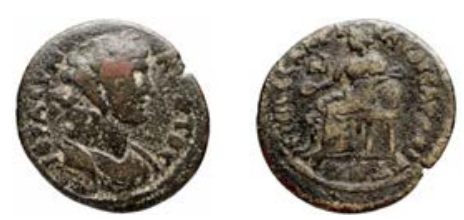

Les données métrologiques et typologiques de ces quatre émissions sont rassemblées dans le tableau ci-dessous :
B. MONNAIES AVEC PORTRAIT IMPÉRIAL, MAIS SANS NOM D'ARCHONTE

\section{Septime Sévère}

1a. AE, 18-20 mm, 3,82 g (13). Axe : 6

AY $\mathrm{K} \bullet \Lambda \cdot \mathrm{CE} \bullet-\mathrm{CEOYHPOC-} \cdot \Pi \mathrm{C} \bullet$; tête laurée de Septime Sévère, à dr.

CAIT $\bullet T H-N \Omega N$; Héraclès nu debout de face, tête à g., sa main dr. posée sur sa massue, la léontè sur son bras $\mathrm{g}$.

1. Londres BMC 44, 2,81; 2. Cambridge McClean 8704 (et Pl. 305. 13), 3,89 ; 3. Paris FG 1067A, 3.74 ; 4. Copenhague $S N G 405,3,27$; 5 . Munich $S N G$ 442, 4,04; 6 . Vienne SNG Leypold 1161, 4,00; 7. Winterthur 3892, 3,$22 ; 8$. New York 1944.100.49386, 3,80 ; 9. NewYork 1988.18.66 (ex Lindgren III, 510), 3,18; 10. von Aulock SNG 3097, 3,61; 11. Tatiş 707, 3,6; 12. CNG EA 349, 22 Avril 2015, lot 275, 4,34; 13. Collection CGT, 2,86.

\begin{tabular}{|c|c|c|c|c|c|}
\hline & $45 \mathrm{~mm}$ & $39-40 \mathrm{~mm}$ & $34-37 \mathrm{~mm}$ & $28 \mathrm{~mm}$ & $22-25 \mathrm{~mm}$ \\
\hline $\begin{array}{l}\text { Attikos } \\
\text { premier } \\
\text { archonte pour } \\
\text { la deuxième fois }\end{array}$ & & & $\begin{array}{l}\text { Septime Sévère } \\
\text { Asclépios, } \\
\text { Hygie et Télesphore } \\
26,83 \text { (4) }\end{array}$ & $\begin{array}{l}\text { Julia Domna } \\
\text { temple tétrastyle } \\
\text { d'Aphrodite Cnidia; } \\
\text { Héraclès } \\
13,69(8)\end{array}$ & $\begin{array}{l}\text { Sans portrait impérial } \\
\text { Cybèle; } \\
\text { Apollon } \\
6,60(31)\end{array}$ \\
\hline $\begin{array}{l}\text { Andronikos } \\
\text { premier } \\
\text { archonte }\end{array}$ & & & $\begin{array}{l}\text { Julia Domna } \\
\text { Mên } \\
\\
24,23(6)\end{array}$ & $\begin{array}{l}\text { Albin César } \\
\text { Zeus Lydios; } \\
\text { Aphrodite Cnidia; } \\
\text { temple tétrastyle } \\
\text { d'Aphrodite Cnidia } \\
11,89 \text { (14) }\end{array}$ & $\begin{array}{l}\text { Septime Sévère } \\
\text { Mên ; } \\
\text { Tychè ; } \\
\text { Apollon } \\
8,14 \text { (39) }\end{array}$ \\
\hline $\begin{array}{l}\text { Andronikos } \Delta \\
\text { premier } \\
\text { archonte }\end{array}$ & $\begin{array}{l}\text { Septime Sévère } \\
\text { Mên et Cybèle } \\
44,29 \text { (3) }\end{array}$ & $\begin{array}{l}\text { Julia Domna } \\
\text { Cybèle } \\
32,73 \text { (1) }\end{array}$ & & & \\
\hline $\begin{array}{l}\text { Chariclès } \\
\text { premier } \\
\text { archonte pour } \\
\text { la deuxième fois }\end{array}$ & 更 & 更 & \begin{tabular}{|l} 
Julia Domna \\
empereur à cheval ; \\
Asclépios, \\
Hygie et Télesphore \\
Caracalla Auguste \\
empereur à cheval, \\
Asclépios, \\
Hygie et Télesphore \\
22,49 (5)
\end{tabular} & $\begin{array}{l}\text { Caracalla Auguste } \\
\text { Héraclès ; } \\
\text { temple tétrastyle } \\
\text { d'Aphrodite Cnidia } \\
\\
\end{array}$ & $\begin{array}{l}\text { Sans portrait impérial } \\
\text { Cybèle }\end{array}$ \\
\hline
\end{tabular}

Fig. 1: Données métrologiques et typologiques de les émissions avec nom d'archonte. 


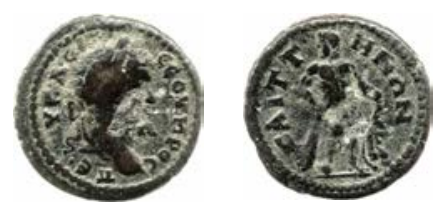

1b.AE, $18 \mathrm{~mm} .3,86 \mathrm{~g}(6)$. Axe : 6

AY $\mathrm{K} \bullet \Lambda \bullet \mathrm{CE} \bullet-\mathrm{CEOYHPOC-} \bullet \Pi E \bullet ;$ tête laurée de Septime Sévère, à dr.

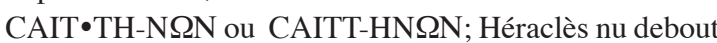
de face, tête à dr., la léontè sur son bras dr., sa main g. posée sur sa massue.

1. Numismatik Naumann 44, 7 Août 2016, lot 626, 3.79 ; 2-6. Collection CGT, 4,76, 4,05, 3,86, 3,54, 3 , 13.
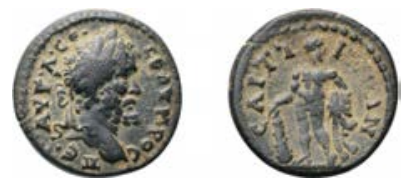

2a. AE, $18-20 \mathrm{~mm}, 3,76 \mathrm{~g}(5)$. Axe : 6

AY $\Lambda \cdot \mathrm{CE} \Pi \bullet \cdot \bullet^{-C E O Y H P O C-~} \bullet$ EP $\bullet$; tête laurée de Septime Sévère, à dr.

CAIT•TH-N $\Omega N$; Héraclès nu debout de face, tête à g., sa main dr. posée sur sa massue, la léontè sur son bras $\mathrm{g}$.

1. Paris FG 1067, 2,97 ; 2. Lanz 117, 24 Novembre 2003 , lot $874,3,99$; 3. Gitbud et Naumann 19, 6 Juillet 2014, lot 402, 3,87; 4. Gitbud et Naumann 34, 2 Août 2015, lot 680, 4,45; 5. Collection CGT (ex London Ancient Coins 39, 18 Novembre 2014, lot 100), 3,52 .
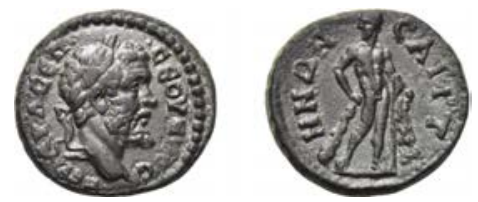

2b.AE, $18-20 \mathrm{~mm}, 4,30 \mathrm{~g}(2)$. Axe : 6

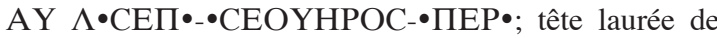
Septime Sévère, à dr.
CAIT•TH-N $\Omega N$; Héraclès nu debout de face, tête à dr., la léontè sur son bras dr., sa main g. posée sur sa massue.

1. Copenhague $S N G$ 406, 2,86 ; 2. CNG 90, 23 Mai 2012, lot 1001, 5,75.

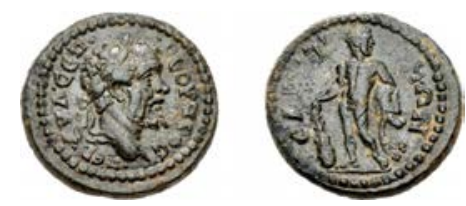

3. AE. 17-18 mm, 2,84g (3). Axe: 6

AY $\Lambda \cdot \mathrm{CE} \bullet \bullet-C E O Y H P O C-\bullet \Pi E P$; tête laurée de Septime Sévère, à dr.

CAIT $\bullet T H-N \Omega N$; Tychè debout de face, tête à g., tenant gouvernail et corne d'abondance.

1. Numismatik Naumann 39, 3 Janvier 2016, lot 630 (ex Gorny et Mosch 233, 6 Octobre 2015, lot 1988), 3,05 ; 2. Numismatik Naumann 52, 2 Avril 2017, lot 326, 2,75; 3. Collection CGT, 2,71 .
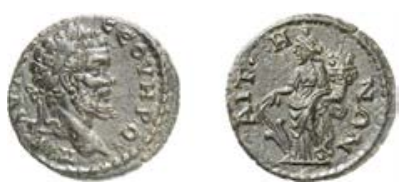

Julia Domna

1. AE. $20 \mathrm{~mm}, 3,40 \mathrm{~g}(4)$. Axe : 6

IOYAI-A CEBAC ; buste drapé de Julia Domna à dr. CAITTH-NSN; Tychè debout de face, tête à g., tenant gouvernail et corne d'abondance.

1. Vienne SNG Leypold 1163, 2,85;2. New York 1998.18.67 (ex Lindgren III, 511), 3,10 ; 3-4, Collection CGT, 3,88, 3,76.

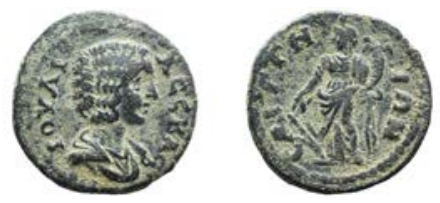


2. AE. $20 \mathrm{~mm} \cdot 4,10 \mathrm{~g}(11)$. Axe : 12,6

IOY $\Lambda$ I-A CEBAC ; buste drapé de Julia Domna à dr. CAIT-THN $\Omega N$; Asclépios debout de face, regardant vers la g., sa main dr. appuyée sur un bâton autour duquel est enroulé un serpent.

1. Londres $B M C 45,2,95$; 2. Copenhague $S N G 407,4,86$; 3. NewYork 1944.100.49369, 3,80; 4. von Aulock $S N G$ $8248,4,86$; 5. Lindgren 791, 3,57 ; 6-8. Tatiş 710-2, 4,52, 5,67, 4,46; 9. Peus 403, 27 Avril 2011, lot 679, 3,$93 ; 10-11$. Collection CGT, 3,97, 2,53.
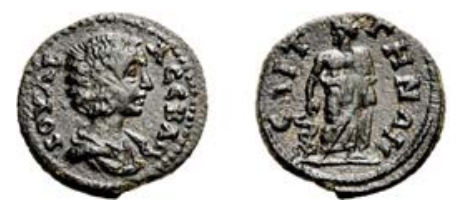

3. AE. $20 \mathrm{~mm}, 3,84 \mathrm{~g}(2)$. Axe : 6

IOY $\Lambda \mathrm{I}-\mathrm{A}$ CEBAC ; buste drapé de Julia Domna à dr. CAIT-THN $\Omega N$; Cybèle assise à g., coiffée du polos, tenant une phiale dans sa main dr. tendue, son bras g. posé sur un tambourin; à ses pieds, un lion.

1. Londres 1906-6-1-89, 3,75 ; 2. Numismatik Naumann 52, 2 April 2017, lot 1225 (ex Aufhäuser 8, 10 Oct. 1991, lot 461), 3,93.
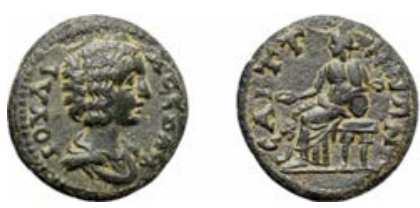

\section{Caracalla}

1. $16 \mathrm{~mm}, 1,94 \mathrm{~g}(1)$. Axe : ?

AY K M AYP - ANTQNEINOC; buste lauré et cuirassé de Caracalla à dr., avec paludamentum

CAITT-HN $\Omega N$; Apollon nu de face, les jambes croisées, tête à g., tenant une branche de laurier dans la main dr., son bras g., duquel tombe un pan de draperie, reposant sur une colonne basse.

1. Weber $6890,1,94$. Seul le revers de cette pièce est illustré Pl. 244. La légende de droit est lue AY K M AYPA - ANTSNEINOC, ce qui n'a guère de sens.
Géta

1. $16 \mathrm{~mm}, 2,12 \mathrm{~g}(6)$. Axe : 6

$\Lambda$ СЕП- ГЕТАС K; buste tête nue et cuirassé de Géta à dr., avec paludamentum, vu de trois quarts en arrière CAITT-HNQN; Apollon nu de face, les jambes croisées, tête à g., tenant une branche de laurier dans la main dr., son bras g., duquel tombe un pan de draperie, reposant sur une colonne basse.

1. Copenhague SNG 409, 2,39 ; 2. Munich SNG 444, 1,93 ; 3. MM Deutschland 12, 10 Avril 2003, lot 201, 1,69 ; 4. Gitbud et Naumann 37, 1 Nov. 2015, lot 499, 2,04; 5-6. Collection CGT, 3,01, 1,64.
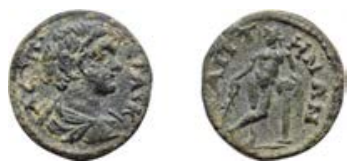

\section{COMMENTAIRE}

\section{CHRONOLOGIE DES ÉMISSIONS}

Quatre émissions signées par des archontes ont été frappées aux noms de Septime Sévère et Julia Domna ; Clodius Albinus est également représenté sur celle d'Andronikos premier archonte, ce qui fournit une fourchette chronologique assez précise pour cette émission : Albin a été nommé César durant le second semestre 193, après le 10 septembre, peut-être même à la fin de l'année en même temps qu'il était désigné consul pour 194 avec Septime-Sévère ${ }^{18}$ et il est déclaré ennemi public par le Sénat vers le 15 décembre 195 , après avoir pris le titre d'Auguste ${ }^{19}$. L'émission d'Andronikos date donc de 194/5. La cité de Saitta n'a pas été la seule dans la province d'Asie à représenter Albin sur son monnayage, puisque l'on connaît des frappes à son nom à Smyrne ${ }^{20}$ et à Sardes ${ }^{21}$.

Quant à celle de Chariclès, elle associe Caracalla Auguste et Géta César à leurs parents : elle a donc été frappée après le 28 janvier 198 quand Septime Sévère proclama la conquête du royaume parthe et prit le titre que Trajan avait le premier porté, celui de 'Parthicus Maximus' ${ }^{22}$. Il nomma alors Caracalla, alors âgé de 9 ans $^{23}$, Auguste et son frère cadet Géta César ${ }^{24}$. 
Restent Attikos et Andronikos $\Delta$ premier archonte. La place d'Attikos dans la série semble s'imposer d'ellemême en tête : elle partage avec Andronikos les mêmes dénominations (34-37 mm, $28 \mathrm{~mm}$ et $22-25 \mathrm{~mm}$ ), mais qui sont plus lourdes, le portrait de Septime Sévère est un portrait de début de règne et la titulature longue de Julia Domna (IOY $\Lambda$ IA $\triangle$ OMNA CEBACT) témoigne elle aussi du début du règne : elle sera réduite, comme à Rome et Laodicée, en IOYAIA CEBACTH, dès l'émission d'Andronikos. L'émission d'Attikos peut donc être datée de 193 ou à la rigueur de 194, si l'on date celle d'Andronikos de 195. Comme Attikos est premier archonte pour la deuxième fois, il est probable qu'il a exercé sa première charge sous le règne de Commode.

Reste le cas d'Andronikos $\Delta$ premier archonte, dont la titulature pose problème. Imhoof-Blumer, en publiant son exemplaire qui devait entrer dans les collections de Berlin (III, 1, 1), en donnait la lecture suivante ${ }^{25}$ : EПI AN $\Delta$ PONEIKOY $\Delta \mathrm{IO} \Delta \Omega \mathrm{P}[\quad]$. H. Bloesch, sur l'exemplaire de Winterthur (III, 1, 2), lisait $\Delta \mathrm{IO} \Delta \Omega \mathrm{P}[]^{26}$, faisant fi du point séparatif entre $\Delta$ et $\mathrm{IO}[. . .$.$] et cette lec-$ ture fut acceptée par E. Lane ${ }^{27}$. Dans le manuscrit publié après sa mort, $\mathrm{H}$. Bloesch était revenu sur cette lecture et la donnait telle que je l'ai acceptée, car celle-ci a depuis été confortée par l'exemplaire de Julia Domna (III, 2, 1), apparue sur le marché en 2000. Mais son interprétation reste difficile. H. Bloesch la comprend ainsi : EПI AN $\triangle$ PONEIKOY $\Delta(\mathrm{IC})$ IO $\Lambda \Lambda$ A K(PATICTOY) CТЕФA(NHФOPOY) APX(ONTOC) A, Andronikos, fils de Iollas $^{28}$, kratistos ${ }^{29}$ stéphanéphore, premier archonte pour la deuxième fois. On pourrait l'interpréter différemment : Andronikos, quatrième du nom $(\Delta)$, fils de Iollas, kratistos stéphanèphore, premier archonte ou Andronikos, pour la deuxième fois, fils de Iollas et/K(AI) Stepha(nos/nas) premier archonte ${ }^{30}$. La première interprétation se heurterait au fait qu'Andronikos, qui signe deux émissions sous Sévère, ne mentionnerait sur aucune des deux un archontat pour la deuxième fois, sauf à estimer que nous avons affaire à deux Andronikos, dont l'un précise sa filiation ; quant à la seconde, elle ne trouve aucun parallèle à Saïtta pour un double archontat.

Il me semble somme toutes que l'interprétation 'Andronikos, quatrième du nom $(\Delta)$, fils de Iollas, kratistos stéphanèphore $^{31}$, premier archonte' est la bonne pour deux raisons : tous les archontes qui ont exercé une seconde fois la charge de premier archonte, et qui ont frappé monnaie, l'indiquent par la formule TO B ; pourquoi Andronikos, fils de Iollas, dérogerait-il à la règle et utiliserait $\mathrm{t}$-il la formule $\Delta(\mathrm{t} \varsigma)^{32}$ ? APX $\cdot \mathrm{A} \bullet$ vient après son nom et son patronyme. Mais, dans un cas, le graveur semble s'être rendu compte qu'il n'avait plus de place dans la légende qui court autour du type de Mên et Cybèle et a placé cette indication, très curieusement, après l'ethnique, alors qu'il aurait pu la placer dans le champ à gauche, comme il l'a fait sur la monnaie de Julia Domna, dans le champ à droite.

Andronikos $\Delta$, fils de Iollas, émet un monnayage rare, connu de nos jours par quatre exemplaires (III, 1 et 2). C'est certainement lui qui introduit les nouvelles dénominations de $45 \mathrm{~mm}$ et $39-40 \mathrm{~mm}$, qui seront reprises par Chariclès et complétées des anciennes. Son émission se situe donc en 196/7.

Quant aux monnaies divisionnaires sans nom d'archonte, il est difficile de les attribuer à tel ou tel archontat. Elles semblent représenter trois dénominations :

\begin{tabular}{|l|c|c|c|}
\hline & $18-20 \mathrm{~mm}$ & $17-18 \mathrm{~mm}$ & $16 \mathrm{~mm}$ \\
\hline Septime Sévère & $3,85(26)$ & $2,84(3)$ & \\
\hline Julia Domna & $3,90(17)$ & & \\
\hline Caracalla Auguste & & & $1,94(1)$ \\
\hline Géta César & & & $2,12(6)$ \\
\hline
\end{tabular}

Tout au plus peut-on affirmer que les séries de Caracalla et de Géta ne peuvent être antérieures à l'archontat de Chariclès. Mais rien n'oblige à lier ces séries aux émissions signées par des archontes. L'atelier de Saittai a $\mathrm{pu}$ vouloir approvisionner régulièrement en menue monnaie les circuits d'échanges locaux.

\section{STRUCTURE DES ÉMISSIONS ET RÉPERTOIRE TYPOLOGIQUE}

La structure de certaines émissions est parfois étonnante, en particulier celle d'Andronikos. On s'attendrait à ce que Septime Sévère figure sur la dénomination la plus large (34-37 mm), et Julia Domna sur la dénomination la plus petite (22-25 mm), Albin César occupant la dénomination intermédiaire (28) mm). Mais c'est l'inverse qui se produit : Julia Domna est représentée sur la plus lourde et Septime Sévère sur la plus légère, sans que l'on puisse comprendre les raisons de cette inversion de préséance. Quant à l'émission de Chariclès, forte de quatre dénominations (plus une cinquième, la plus petite, dédiée, comme souvent, à des monnaies sans portrait impérial), on s'attendrait à l'ordre Septime Sévère, Caracalla Auguste, Géta César et Julia Domna (ou Julia Domna 
et Géta César), mais c'est Géta (et non Caracalla) qui est représenté sur la dénomination de 39-40 mm, Caracalla frappant aussi bien des dénominations de 34-37 mm (avec sa mère) et de $28 \mathrm{~mm}$. Là encore la logique de ce choix de l'atelier de Saittai nous échappe.

Le répertoire iconographique est le suivant :

\begin{tabular}{|l|c|c|c|c|}
\hline & Attikos & Andronikos & Andronikos $\Delta$ & Chariclès \\
\hline $\begin{array}{l}\text { Aphrodite } \\
\text { Cnidia }\end{array}$ & & $\mathrm{x}$ & & \\
\hline $\begin{array}{l}\text { Aphrodite } \\
\text { Cnidia, temple }\end{array}$ & $\mathrm{x}$ & $\mathrm{x}$ & & $\mathrm{x}$ \\
\hline Apollon & $\mathrm{x}$ & $\mathrm{x}$ & & \\
\hline $\begin{array}{l}\text { Asclépios, } \\
\text { Hygie et } \\
\text { Téléphore }\end{array}$ & $\mathrm{x}$ & & & $\mathrm{x}$ \\
\hline Cybèle & $\mathrm{x}$ & & $\mathrm{x}$ & $\mathrm{x}$ \\
\hline $\begin{array}{l}\text { Empereur à } \\
\text { cheval }\end{array}$ & & & & $\mathrm{x}$ \\
\hline Héraclès & $\mathrm{x}$ & & & $\mathrm{x}$ \\
\hline $\begin{array}{l}\text { Héraclès et } \\
\text { Géryon }\end{array}$ & & & & $\mathrm{x}$ \\
\hline Mên & & $\mathrm{x}$ & & \\
\hline Mên et Cybèle & & & $\mathrm{x}$ & $\mathrm{x}$ \\
\hline Tychè & & $\mathrm{x}$ & & \\
\hline Zeus Lydios & & $\mathrm{x}$ & & \\
\hline
\end{tabular}

Un type n'est pas lié spécifiquement à une dénomination, encore que le temple d'Aphrodite Cnidia se rencontre uniquement sur celle de $28 \mathrm{~mm}$. Ce répertoire n'est pas entièrement nouveau, puisque Mên, Cybèle, Héraclès et Apollon figuraient déjà sur le monnayage de Marc Aurèle et Lucius Verus ${ }^{33}$; les types d'Aphrodite Cnidia et du temple d'Aphrodite seront introduits sous Commode $^{34}$. Il convient de noter l'inflexion donnée par Saittai à son iconographie à partir du règne joint de Marc Aurèle et Lucius Verus. Sous Hadrien, les revers sont d'essence romaine, avec Tychè serrant la main de l'empereur Hadrien ${ }^{35}$ ou Roma assise tenant une Nikè ${ }^{36}$. Il est possible du reste que ces types fassent référence à une visite d'Hadrien à Saittai en $124^{37}$. A partir de la deuxième moitié du IIe siècle, les monnayages civiques deviennent la marque de l'identité culturelle hellénique et ce changement de perspective aura du reste été encouragé par Hadrien lui-même, infatigable voyageur qui aura parcouru toutes les provinces (ou presque) de son Empire. Outre les divinités panhelléniques classiques ${ }^{38}$, Apollon, Asclépios et Hygie et Héraclès, Saittai fait évidemment représenter Zeus Lydios, mais surtout les dieux phrygiens Mên ${ }^{39}$ et Cybèle, dont les cultes étaient extrêmement populaires en Asie Mineure. La statue d'Aphrodite Cnidia, l'œuvre de Praxitèle, seule ou dans son temple, est un thème plus rare ${ }^{40}$, mais constant à Saittai, puisqu'on le retrouvera sous Alexandre Sévère ${ }^{41}$, Gordien $\mathrm{III}^{42}$, Philippe $^{43}$ et Gallien ${ }^{44}$. On notera que ce type est plus souvent associé à une impératrice (Crispine, Julia Domna, Tranquiline, Otacilie, Salonine) qu'à un empereur (Albin César, Caracalla Auguste et Alexandre Sévère). La représentation d'Héraclès tuant Géryon, le géant à trois corps, pour lui voler son troupeau de bœufs, associé à Géta César sur l'émission de Chariclès, est quant à elle extrêmement rare ${ }^{45}$.

Il faut conclure. Le monnayage de Saittai frappé sous Septime Sévère et sui pose un certain nombre de problèmes chronologiques et onomastiques que j'espère avoir résolus. Saittai était sous l'Empire une cité modeste et son monnayage intermittent de peu d'importance. La fonction de ces frappes n'est évidemment pas économique, mais c'est une affaire de prestige. Les cités grecques entendent montrer que leur identité est irréductible, tout en s'accoutumant au pouvoir romain.

\section{NOTES}

1. Monnaie acquise de Kovacs MBS XV, 1 Oct. 2003, lot 244 ; ici IV, 1, 1 .

2. Nathan Elkins, maintenant Assistant Professor of Art History à Baylor University, avait participé en 2004 au Summer Seminar organisé par l'American Numismatic Society dont j'étais le Visiting Scholar. Il était alors à l'Université du Missouri et c'est lui qui me fit connaître cette acquisition, ce dont je le remercie chaleureusement.

3. Saittai est une cité lydienne, située dans la haute vallée de l'Hermos. Elle faisait partie du conventus de Sardes. Saittai (de nos jours Sidas kale) est située entre les villes modernes de Demirci et Kula.

4. Lawrence 2008. Je dois également la connaissance de cet article à N. Elkins.

5. P. Lawrence s'intéresse essentiellement à l'iconographie de Mên et de Cybèle. Son article est du reste dédié à la mémoire d'E. N. Lane qu'elle avait connu à Athènes, lorsqu'elle était membre de l'American School of Classical Studies de 1959 à 1961 .

6. D'autant que le Roman Provincial Coinage V, consacré aux règnes de Septime Sévère et sui et de Macrin (193-218), est en préparation. Saittai a émis du numéraire à époque impériale sous Hadrien, puis de façon presque continue à partir du règne joint de Marc Aurèle et Lucius Verus jusque sous Gallien (on ne connaît pas de monnayage pour la période 249-253, ni pour Valérien). Pour les monnayages d'Hadrien, des Antonins, d'Elagabale, de Maximin et de Gordien III, voir le RPC Online.

7. Ce catalogue est typologique. Il ne s'agit pas d'une étude de coins, même si des identités de coin ont pu être notées. Les points séparatifs dans les légendes sont indiqués, mais il se 
peut qu'ils n'aient pas tous été vus, en raison de l'état de conservation des pièces. Mes remerciements vont à $\mathrm{A}$. Burnett (Londres), K. Dahmen (Berlin), J. Mairat (Oxford) D. Rousset (Paris), Ch. Schinzel (Winterthur), K. Vondrovec (Vienne) et B. Zäch (Winterthur) pour leur aide.

8. Même coin de droit que Héraclée (Kraft Taf. 77, 2b).

9. La légende de revers a été lue EПI TOY $\Lambda$ IOY APX A TO B par Mionnet $1809,112, \mathrm{n}^{\circ} 619$, créant ainsi un nom d'archonte qui n'existe pas. Il faut donc le supprimer de Münsterberg 1973, 146 et Leschhorn 2009, 850 (mais qui doutait de ce nom : 'Auflösung des Namens unsicher').

10. Même coin de droit que Attuda, Héraclée, Laodicée, Maionia (Kraft 1972, Taf. 79, 21a-d).

11. Monnaie citée deux fois comme Paris, BnF 41784077 (Martin 2013, Saïttai 7) et comme Paris Wadd. 5167 (Martin 2013, Saïttai 22).

12. Cette monnaie illustrée dans Martin 2013, Taf. 14 sous le ${ }^{\circ}$ Saittai 3/17.

13. Liaison de coin de droit avec Gordos (Kraft 1972 : Taf. 69, $50 \mathrm{~b})$.

14. Le site web du MFA ne connaît pas le pedigree de cette pièce.

15. University of Missouri. Museum Magazine, Museum of Art and Archaeology 45 (Fall 2004), 11, figs. 3 et 3a ; Recent Acquisitions, Muse 36-38 (2002-2004), 94.

16. Même coin de droit que Sardes (Kraft 1972 : Taf. 70, 58a).

17. Forni $1994,142-3, n^{\circ} 334$.

18. Daguet-Gagey 1998.

19. Daguet-Gagey $2000: 229-31,257-9,261-74$; Birley 1996 ; Lusnia 2013.

20. BMC Ionia, 281-2/360-2.

21. BMC Lydia, 259/146; MM Deutschland 15, 21 Octobre 2004 lot 845 ; Numismatik Naumann 44, 7 Juin 2016, lot 631 (ex Finarte 995, collection Fontana, 26 Novembre 1996, lot 993 ; Helios 5, 25 Juin 2010, lot 853).

22. Guey 1948 ; Le Glay ; Voisin ; Le Bohec 1991 : 380 ; DaguetGagey $2000: 290-1$ : exactement cent ans après l'avènement de Trajan, le 28 janvier étant son dies imperii. Mais le 28 janvier 117 n'est pas le jour où Trajan reçut le titre de Parthicus Maximus, même s'il est possible que la chancellerie romaine s'employa à faire coïncider les chronologies (Daguet-Gagey 2000 : 290 : Il était facile de rapprocher Septime Sévère de son illustre ancêtre, dont il se considérait comme l'arrière-arrière-petit-fils...Désormais Sévère pouvait faire figurer dans sa titulature officielle le titre de Parthicus Maximus, qui achevait de consacrer son rapprochement avec la dynastie antonine). Trajan obtint ce titre dès février 116 (20 ou 21 ?) (Woytek $2010: 16-17)$.

23. Caracalla était né à Lugdunum (Lyon) le 4 avril 188 .

24. Birley 1988 : 130. Géta est né en mai 189, sans doute le 27, à Rome.

25. Imhoof-Blumer $1913: 56, \mathrm{n}^{\circ} 161$.

26. Bloesch $1964: 63, \mathrm{n}^{\circ} 434$ et Pl. 23

27. Lane $1975: 38, \mathrm{n}^{\circ} 10$.

28. Le nom de Iollas est très fréquent en Lydie : $L G P N$ V.A, p 225-6 ; du reste un Iollas (Aurelios Septimios Iollas) est également archonte à Saitta sous Philippe : Winterthur 3897 ; Helios Numismatik 3, 29 Avril 2009, lot 503.

29. Imhoof-Blumer 1897, 142, $\mathrm{n}^{\circ} 21$ : sur une monnaie de Sardes frappée sous Gallien, la mention de kratistos (KPAT) archiereus.

30. Je dois ces différentes suggestions à la regrettée Elaine Matthews (LGPN Oxford) et à Édouard Chiricat (LPGN Oxford) qui m'ont fait profiter de leur science, ce dont je leur suis reconnaissant.
31. La fonction de stéphanèphore n'existe du reste pas à Saittai. On en trouve une mention en 101/2 dans l'agglomération (katoikia) de Koloe, proche de Saittai (TAM V, 1, 193 ; TAM V, 1, 192). Dans l'Est de la Lydie, de la Mysie et dans certaines cités de Phrygie, la principale magistrature est celle de premier archonte : RPC VII, 1,46 et 312 , carte 4 . On trouve toutefois des stéphanèphores à Maeonia, cité lydienne proche de Saittai : sous Caracalla (BMC Lydia, 134/48) et Trajan Dèce (RPC IX, $\left.\mathrm{n}^{\circ} 725\right)$. Dans les deux cas, les magistrats sont APX A TO B K CTEФANH(ФOPOY), le K se comprenant KAI. Si Andronikos avait été premier archonte et stéphanèphore, К СТЕФA devrait venir avant APX A.

32. Dont on ne connaîtrait que de rare parallèle : un seul cas, quand on interroge $R P C$ Online, à Magnésie du Méandre, $\triangle \mathrm{IC}$ semblant du reste s'appliquer au magistrat Moschiôn (Moschiôn deuxième du nom) (RPC VI Online 5227). La lecture ЕПI ГР $\triangle \mathrm{IC} \mathrm{K}$ Z $\Omega C I M O Y$ sur une monnaie de Gordien III à Tralles conservée à Tübingen (SNG Tübingen 5. Heft. Karien und Lydien, $\mathrm{n}^{\circ} 3871$ ) ne peut être acceptée (voir RPC VII, $1, \mathrm{n}^{\circ}$ 507).

33. Mên : RPC IV Online 1391 (Marc Aurèle), 6957 (Lcius Verus); Cybèle : RPC IV Online 4806 (Marc Aurèle) ; Héraclès : RPC IV Online 1390 (Marc Aurèle) ; Apollon : RPC IV Online 11061 (Faustine II).

34. $R P C$ IV Online 1395, 1397 (Crispine).

35. RPC III, 2543A.

36. $R P C$ III, 2543. On retrouve cependant ce type sous Marc Aurèle (RPC IV Online 1389).

37. Weiss 1995. Toutefois Dionysos figure au revers de Sabine (RPC III, 2544). Cette divinité ne sera représentée ensuite que sur des monnaies sans portrait impérial ( $R P C$ IV Online 9961 ; $R P C$ VI Online 4427 ; $R P C$ VII, 1, 229).

38. On notera toutefois un thème 'romain' dans l'émission de Chariclès : l'empereur à cheval terrassant un ennemi (associé à Julia Domna et Caracalla), évoquant sans doute la conquête du royaume parthe.

39. L'inscription d'un autel du IIIe siècle à Saittai le qualifie de « dieu unique, immortel » : Robert 1987 : 427 et n. 1 avec bibliographie antérieure.

40. On le trouve à Laodicée de Phrygie sous Antonin (RPC IV Online 2092 : Marc Aurèle César), à Nicée sous Lucius Verus (RPC IV Online 5972), à Philadelphie et Alioi sous Marc Aurèle $(R P C$ IV Online 1371 et 1762 : Faustine II) ; à Cnide, sous Septime Sévère : Gorny and Mosch, e-auction 251, 15 Octobre 2017, lot 4676 (Caracalla Auguste et Plautilla).

41. $R P C$ VI Online 4443.

42. RPC VII, 1, 224 : Tranquilline.

43. BMC Lydia 225/65 Otacilie.

44. BMC Lydia 226/74 : Salonine.

45. Egalement à Blaundos : Voegtli 1977 : 37 et Taf. 6a-b. Les représentations les plus courantes montrent Héraclès s'emparant des bœufs du troupeau de Géryon : Voegtli 1977 : 3840 et Taf. $6 c-f$.

\section{BIBLIOGRAPHIE}

AMANDRY, M. (2017) : Quelques témoignages numismatiques de cités de la province d'Asie après l'élévation de Caracalla à l'Augustat et de Géta au Césarat en 198, BSFN 72, $5,137-43$.

BIRLEY, A. (1988) : The African Emperor Septimius Severus, Londres. 
BIRLEY, A. (1996) : Clodius Septimius Albinus Decimus, The Oxford Classical Dictionary (S. Hornblower, A. Spawforth, eds), Oxford, 351.

BLOESCH, H. (1964) : Antike Kleinkunst in Winterthur. II. Münzen, Winterthur.

BMC Ionia $=$ HEAD, B. V. (1892) : A Catalogue of the Greek Coins in the British Museum. Greek Coins of Ionia, Londres.

BMC Lydia = HEAD, B. V. (1892) : A Catalogue of the Greek Coins in the British Museum. Greek Coins of Lydia, Londres.

DAGUET-GAGEY, A. (1998) : Adrastus et la colonne antonine. L'administration des travaux publics à Rome en 193 ap. J.-C., MEFRA 110, 893-915, plus part. 912-4.

DAGUET-GAGEY, A. (2000) : Septime Sévère. Rome, l'Afrique et l'Orient, Paris.

FORNI, G. (1994) : IEPA e $\Theta E O \Sigma \Sigma$ YNK $\Lambda$ HTO $\Sigma$. Un capitolo dimenticato nella storia del senato romano, Scritti vari di storia, epigrafia e antichità romane (M. G. Angeli Bertinelli , ed), Rome, 51-187 (= Memorie dell'Academia Nazionale dei Lincei, cl. sc. mor., s. 8, V 3 (1953), 49-168).

GUEY, J. (1948) : 28 janvier 98-28 janvier 198, ou le siècle des Antonins, REA 50, 60-70.

IMHOOF-BLUMER, F. (1897) : Lydische Stadtmünzen, Genf/ Leipzig.

IMHOOF-BLUMER, F. (1913) : Antike grieschische Münzen, RSN XIX, 5-134 et Pl. I-IV.

KRAFT, K. (1972) : Das System der Kaiserzeitlichen Münzprägung in Kleinasien, Berlin.

LANE, E. (1975) : Corpus Monumentorum Religionis Dei Menis (CMRDM), II, The Coins and Gems, Leiden.

LAWRENCE, P. (2008) : A Bronze Septimius Severus at Saitta, Issued by Charikles. An Avatar or Acolyte for the Moon-God Men?, Muse 42, 31-42.

LE GLAY, M. ; VOISIN, J.-L. ; LE BOHEC, Y. (1991) : Histoire romaine, Paris.

LESCHHORN, W. (2009) : Lexikon der Aufschriften auf griechischen Münzen II Ethnika und 'Beamtennamen', Vienne.

$L G P N=$ CORSTEN, T., ed (2010) $:$ A Lexicon of Greek Personal Names V.A. Coastal Asia Minor : Pontos to Ionia, Oxford.
LUSNIA, S. S. (2013) : Clodius Albinus, Decimus, The Encyclopedia of Ancient History (R. S. Bagnall, ed), Oxford, (https:// www.researchgate.net/publication/261615414_Clodius_ Albinus_Decimus).

MARTIN, K. (2013) : Demos. Boule. Gerousia. Personifikationen Städtischer Institutionen auf Kaiserzeitlichen Münzen aus Kleinasien, Teil 1-2, Bonn.

MIONNET, T. E. (1809) : Description de médailles antiques, grecques et romaines IV, Paris.

MÜNSTERBERG, R. (1973) : Die Beamtennamen auf den griechischen Münzen, Hildesheim/New York (réimpression de ses quatre articles rédigés entre 1911 et 1927, le dernier posthume, parus dans la Numismatische Zeitschrift à Vienne).

ROBERT, L. (1987) : Documents d'Asie Mineure, BEFAR 239 bis, Paris.

RPC III = AMANDRY, M. ; BURNETT, A. et al. (2015) : Roman Provincial Coinage III. Nerva, Trajan and Hadrian (AD 96-138), Londres/Paris.

RPC VII, 1 = SPOERRI BUTCHER, M. (2006) : Roman Provincial Coinage VII. De Gordien Ier à Gordien III. 1. Province d'Asie, Londres/Paris.

RPC IX = HOSTEIN, A. ; MAIRAT, J. (2016) : Roman Provincial Coinage IX. From Trajan Decius to Uranius Antoninus (AD 249-254), Londres/Paris.

RPC Online IV = HEUCHERT, V. ; HOWGEGO, C.: Roman Provincial Coinage IV. Antoninus Pius to Commodus (AD 138-192), Oxford (http://rpc.ashmus.ox.ac.uk).

RPC Online VI = CALOMINO, D. ; BURNETT, A.: Roman Provincial Coinage VI. Elagabalus to Maximinus (AD 218238), Oxford (http://rpc.ashmus.ox.ac.uk).

TAM = HERRMANN, P. (1981) : Tituli Asiae Minoris V. Tituli Lydiae linguis Graeca et Latina conscripti, fasc. 1. Regio Septentrionalis ad Orientem Vergens, Vienne.

VOEGTLI, H. (1977) : Bilder der Heldenepen in der Kaiserzeitlichen Griechischen Münzprägung, Aesch.

WEISS, P. (1995) : Hadrian in Lydien, Chiron 25, 213-24.

WOYTEK, B. (2010) : Die Reichsprägung des Kaisers Traianus (98-117), Vienne. 